\title{
Representações sociais de profissionais sobre suas práticas educativas em saúde para mães de prematuros
}

\author{
Social representations of professionals about their health educational practices for preterm \\ infant's mother
}

Representaciones sociales de profesionales sobre sus prácticas educativas en salud para madres de bebés prematuros

Sumaya Medeiros Botêlho ${ }^{1 *}$, Rita Narriman Silva de Oliveira Boery ${ }^{1}$, Washington da Silva Santos ${ }^{1}$, Luciana Araújo dos Reis ${ }^{1}$, Leila Graziele de Almeida Brito ${ }^{1}$, Isnara Teixeira de Britto ${ }^{1}$.

\begin{abstract}
RESUMO
Objetivo: Analisar as Representações Sociais de profissionais sobre suas práticas educativas em saúde para as mães de bebês prematuros hospitalizados. Métodos: Foram utilizados três métodos na coleta de dados: a observação sistemática, o teste de associação livre de palavras e a entrevista semiestruturada. Os dados da observação sistemática e da entrevista semiestruturada foram analisados através da Análise de Conteúdo Temática e o teste de associação livre de palavras através do software EVOC; posteriormente foi feita a triangulação dos dados. Resultados: Após a análise dos dados surgiu uma Classe Temática, Educação em saúde para mães de prematuros, e cinco Categorias: explicações específicas sobre procedimentos realizados; explicações sobre o estado geral do bebê; uso de linguagem acessível; orientações no momento da alta do bebê; fornecimento de um folder educativo no momento da alta do bebê. Conclusão: As representações apreendidas são condizentes com as práticas educativas diárias dos profissionais, que realizam uma assistência de qualidade para os pais na tentativa de diminuir o sofrimento causado pela hospitalização do bebê. Este estudo espera contribuir para o fortalecimento das práticas educativas em saúde capazes de oferecer segurança e habilidade aos pais, com vistas ao cuidado seguro de seu filho prematuro.
\end{abstract}

Palavras-chave: Recém-nascido prematuro, Educação em saúde, Unidades de terapia intensiva neonatal, Orientação.

\begin{abstract}
Objective: To analyze the Social Representations of professionals about their educational health practices for mothers of hospitalized premature babies. Methods: Three methods were used to collect data: systematic observation, the free word association test and the semi-structured interview. Data from systematic observation and semi-structured interviews were analyzed using thematic content analysis and the free word association test using the EVOC software; subsequently the data was triangulated. Results: After the data analysis, a Thematic Class, Health Education for mothers of preterm infants, and five Categories emerged: specific explanations about procedures performed; explanations about the baby's general condition; use of accessible language; guidelines when the baby is discharged; provision of an educational folder at the time of baby's discharge. Conclusion: The representations apprehended are consistent with the professionals' daily educational practices. They provide quality care for parents in an attempt to reduce the suffering caused by the baby's hospitalization. This study hopes to contribute to the strengthening of educational health practices capable of offering parent's safety and ability, with a view to the safe care of their premature child.
\end{abstract}

Keywords: Infant premature, Health education, Intensive care units neonatal, Guidance.

1 Universidade Estadual do Sudoeste da Bahia (UESB), Jequié - BA.

*E-mail:sumayamedeiros@hotmail.com 


\section{RESUMEN}

Objetivo: Analizar las representaciones sociales de profesionales sobre sus prácticas educativas en salud para las madres de bebés prematuros hospitalizados. Métodos: Fueron utilizados tres métodos para la recolección de datos: La observación sistemática, el test de asociación libre de palabras y la entrevista semiestructurada. Los datos de la observación sistemática y de la entrevista semiestructurada fueron analizados através del Análisis de Contenido Temático y el Test de Asociación Libre de Palabras através del software EVOC; posteriormente fue hecha la triangulación de los datos. Resultado: Después del análisis de los datos surgió una Clase Temática, Educación en Salud para Madres de Prematuros, y cinco categorías: Explicaciones específicas sobre procedimientos realizados; explicaciones sobre el estado general del bebé; uso de lenguaje asequible; orientaciones en el momento de dar de alta al bebé. Conclusión: Las representaciones aprehendidas se corresponden con las prácticas educativas diarias de los profesionales, quienes brindan una asistencia de calidad para los padres con el intento de disminuir el sufrimiento causado por la hospitalización del bebé. Este estudio espera contribuir para el fortalecimiento de las prácticas educativas en salud capaces de ofrecer seguridad y habilidad a los padres, con miras al cuidado atento de su hijo prematuro.

Palabras clave: Recién nacido prematuro, Educación en salud, Unidades de cuidado intensivo neonatal, Orientación.

\section{INTRODUÇÃO}

Em 184 países, a taxa de nascimento prematuro varia de $5 \%$ a $18 \%$ dos bebês nascidos. O bebê prétermo é definido como bebês nascidos vivos antes de 37 semanas de gravidez. Essa classificação de prematuridade é baseada na idade gestacional: extremamente prematuro (menos de 28 semanas), muito prematuro (28 a 32 semanas) e pré-termo moderado a tardio (32 a 37 semanas) (WORLD HEALTH ORGANIZATION (WHO), 2018).

Dentre diversos fatores de risco para prematuridade, pode-se destacar alguns que são considerados fatores socioeconômicos (pobreza, analfabetismo, mães jovens, áreas rurais e ausência de acompanhamento pré-natal) e maternos (anemia e pré-eclâmpsia) que estão inter-relacionados com as causas do nascimento prematuro (AHMAD A, et al., 2018).

Diante do nascimento prematuro o bebê tende a ficar hospitalizado por um tempo até conseguir independência na sua adaptação fora do útero. Com isso, a notícia da hospitalização do filho recém-nascido (RN) rompe os sonhos construídos durante todo o período gestacional, fazendo com que a mãe se sinta frustrada, infeliz ou mesmo culpada, na busca de respostas que justifiquem essa situação (VERONEZ M, et al., 2017). Lidar com essa nova rotina para a qual as famílias não estão preparadas, se torna um obstáculo, nascendo sentimentos indesejáveis e desencantadores. Esses sentimentos são demonstrados diariamente, com a chegada dos pais no domicílio, e a impossibilidade de trazer o filho consigo, vivenciando uma sensação de angústia e vazio (MAIA JMA, et al., 2014).

É nesse contexto que a educação em saúde predomina como instrumento essencial no processo de cuidado ao RN. Ao receberem acolhimento dos profissionais de saúde durante a preparação da alta do RN, através de práticas educativas em saúde, os pais adquirem maior desenvoltura e domínio para lidar com os filhos após a alta hospitalar (RIBEIRO JF, et al., 2015).

O planejamento de atividades educativas em saúde deve ser realizado considerando as necessidades dos sujeitos envolvidos, que partam de sua realidade, de suas dúvidas e problemas. Além disso, é fundamental que estratégias pedagógicas sejam pensadas, que oportunizem 0 alcance de conhecimentos e desenvolvimento de novas posturas diante do cuidado, devendo, então, envolver os participantes nas situações (BUGS BM, et al., 2018).

Este estudo tem a Teoria das Representações Sociais (TRS) como seu referencial teórico, na qual busca conhecer as representações presentes, do tempo atual da sociedade, considerando que o conhecimento produzido é relativo a quem fala e de onde fala, a um grupo de pertença, e não ao objeto em si, enfatizando o valor desse grupo (MOSCOVICI S, 2010). 
A representação social é sempre a representação de alguém (sujeito) sobre alguma coisa (objeto). Ela atribui significados ao objeto, proporcionando-lhe uma relação de simbolização e interpretação; e é exposta como uma modelização do objeto, absolutamente legível de vários suportes linguísticos, comportamentais ou materiais, e também serve para agir sobre o mundo e sobre o outro (JODELET D, 2001).

Assim, o objetivo desse estudo é analisar as representações sociais de profissionais sobre práticas educativas em saúde para as mães de bebês prematuros hospitalizados, visto que essas práticas são consideradas relevantes para o cuidado após a alta hospitalar do bebê.

\section{MÉTODOS}

$\mathrm{Na}$ construção desse estudo optou-se por uma abordagem qualitativa, de natureza exploratória, com embasamento teórico fundamentado na TRS e na Teoria do Núcleo Central. Participaram desse estudo 33 profissionais de saúde de nível superior (médicos, enfermeiros e fisioterapeutas) que acompanham bebês prematuros, na Unidade de Terapia Intensiva Neonatal e na Unidade Semi-intensiva de um Hospital Municipal no Sudoeste da Bahia. A coleta de dados foi realizada no período de junho, julho e agosto de 2018 no próprio hospital.

Os critérios de inclusão foram os profissionais de saúde de nível superior (médicos, enfermeiros e fisioterapeutas) que atuam nas duas unidades e que tenham pelo menos seis meses de atuação na unidade; e os de exclusão foram os profissionais de saúde de nível superior (médicos, enfermeiros e fisioterapeutas) que se encontravam de férias ou de licença no período da coleta de dados.

Inicialmente, a pesquisadora realizou a técnica de observação sistemática; em seguida foi realizado o Teste de Associação Livre de Palavras e depois uma entrevista semiestruturada. Assim, para o Teste de Associação Livre de Palavras foi solicitado que os 33 profissionais participantes evocassem cinco palavras que viessem à mente após a questão indutora: associe cinco palavras ao termo "educação em saúde". Em seguida, que eles enumerassem as evocações de acordo com a ordem de importância. A entrevista semiestruturada foi realizada com 14 profissionais, com perguntas acerca da educação em saúde que é realizada nas unidades pesquisadas.

Para a análise dos dados produzidos pela observação sistemática e pelas entrevistas semiestruturadas foi utilizada a Técnica da Análise de Conteúdo Temática (BARDIN L, 2011). Já os resultados obtidos através do Teste de Associação Livre de Palavras foram processados estatisticamente por meio do software Ensemble de Programmes Permettant l'analyse dês Evocations (EVOC) (QUEIROZ MAC, et al., 2015).

Após as três análises foi realizada a triangulação dos dados, considerada uma das ferramentas utilizadas para potencializar a credibilidade do estudo, consistindo na utilização diversificada de fontes de dados, tipos de dados e de métodos (JÚNIOR AFB, et al., 2016). Contudo, cabe destacar que nessa pesquisa a entrevista semiestruturada foi considerada como a principal metodologia utilizada, sendo as outras duas técnicas definidas como complementares.

A pesquisa atendeu em todos os momentos à Resolução 466/2012 do Conselho Nacional de Saúde, que normatiza as pesquisas envolvendo seres humanos, sendo submetido ao Comitê de Ética em Pesquisa da Universidade Estadual do Sudoeste da Bahia (CEP/UESB), sob o Certificado de Apresentação para Apreciação Ética (CAAE) no 83490317.1.0000.0055. Com o objetivo de garantir o anonimato dos participantes, eles foram identificados no estudo por meio de E1 (entrevistado 1) a E14 (entrevistado 14).

\section{RESULTADOS}

Em relação às características sociodemográficas dos profissionais constatou-se que $81,8 \%$ são mulheres; $72,7 \%$ possuem filhos; $48,5 \%$ são fisioterapeutas, $21,2 \%$ médicos e $30,3 \%$ enfermeiros; $33,3 \%$ possuem especialização em UTIN, 15,2\% em pediatria, 12,1\% em UTIN e pediatria, e 39,4\% possuem especializações em outras áreas; quanto ao tempo de serviço nas unidades $51,6 \%$ possuem até 5 anos no hospital, 24,2\% possuem entre 6 e 10 anos, e 24,2\% possuem 11 anos ou mais.

Diante da análise do tipo prototípica realizada por meio dos resultados do teste de associação livre de palavras, a partir do software EVOC versão 2005, observou-se que os 33 profissionais conseguiram 
contemplar a orientação solicitada, pois cada um evocou cinco palavras, perfazendo um total de 165 palavras evocadas, e em seguida enumeraram as palavras segundo ordem de importância, da mais importante para a menos importante. O corpus foi então constituído a partir da reordenação dos termos evocados conforme a ordem de importância apontada pelos sujeitos do estudo.

Dos 165 termos evocados, 91 foram distintos, porém alguns possuíam o mesmo significado, os quais foram uniformizados substituindo termos sinônimos por um único termo dentre os evocados pelos sujeitos (agregação semântica), garantindo que no sentido final fossem processadas pelo software como sinônimos (42). Após a agregação semântica foram gerados 18 termos diferentes entre si.

No entanto, das 165 evocações, e utilizando a Lei de Zipf, foi determinado um ponto de corte na frequência 4 o que implica que quaisquer termos que tiveram uma frequência inferior a este valor foram descartados da análise, o que gerou um aproveitamento de $71,5 \%$ das palavras evocadas (118 palavras), tornando assim a análise mais representativa. A análise gerou os dados necessários para a construção do quadro de quatro casas, sendo que a partir desse quadro observou-se uma frequência média (ponto de corte superior) de ocorrências das palavras igual a 6; a média das ordens médias (RANG) foi 3.

Após a análise das entrevistas semiestruturadas emergiram uma Classe Temática, Educação em saúde para mães de prematuros, e cinco Categorias, 1) Explicações específicas sobre procedimentos realizados;2) Explicações sobre o estado geral do bebê; 3) Uso de linguagem acessível; 4) Orientações no momento da alta do bebê; 5) Fornecimento de um folder educativo no momento da alta do bebê.

\section{Explicações específicas sobre procedimentos realizados}

A primeira categoria demonstra que as representações dos profissionais estão ligadas com as explicações específicas aos pais sobre os procedimentos que são realizados com seus filhos, conforme as falas a seguir.

"Aqui na unidade nós desenvolvemos ações como orientações para as mães ( ) a gente trabalha também com a condição do bebê, a gente explica a ela como que deve ser feito o manuseio dessa mãe com esse bebê" (E2).

"Tudo que é feito em relação à técnica a gente explica a elas o que que a gente tá fazendo, o porque e o que pode ser possível fazer em casa, a hidratação nasal, a gente faz a lavagem do narizinho, a drenagem, então a gente explica pra que ela possa fazer isso em casa" (E12).

Durante a observação sistemática também foi constatado essa preocupação dos profissionais. A fisioterapeuta explica a uma mãe o procedimento que estava realizando com o seu filho.

Diante desses relatos e da observação da rotina, observa-se que os profissionais, percebem a importância de explicações esclarecedoras acerca dos procedimentos que realizam com os bebês, e fazem também orientações e ensinamentos importantes para que as mães consigam dar continuidade às condutas quando estiverem em casa com o filho.

No teste de associação livre de palavras foi constatado que no núcleo central aparece a palavra continuidade, com uma frequência de 6 e ordem de importância de 2,000; e a palavra ensinamento aparece nos elementos de contraste com frequência de 5 e ordem de importância de 1,200. Observa-se, assim, que os profissionais se empenham em oferecer orientações e ensinamentos aos pais com o objetivo de proporcionar uma continuidade do cuidado no domicílio.

\section{Explicações sobre o estado geral do bebê}

A segunda categoria vem expor as representações dos profissionais através de explicações acerca do estado geral em que os bebês se encontram, segundo os relatos seguintes.

"Porque a mãe chega no ambiente que ela não conhece, ela não sabe como é que está a situação do filho dela, então ela precisa ser recepcionada, desde a entrada da UTI até o leito do bebê pra ela saber o que tá acontecendo com o filho dela, qual o estado do filho dela" (E2). 
"Geralmente as mães, quando estão presentes à noite, que a frequência é bem menor em relação ao dia, essas mães muitas vezes chegam ansiosas, inseguras, então eu costumo assim, esclarecer como a criança está, dentro do possível, eu procuro mostrar que o bebê tá ali, tá justamente no lugar certo, tentando tranquilizar no sentido de que, se ele não estivesse na UTI ele estaria mais desconfortável, sem conseguir respirar" (E14).

Durante a observação sistemática também foi verificada essa atitude dos profissionais: A médica e a enfermeira discutem um caso, e em seguida a médica começa a passar os boletins para as mães, fornecendo todas as informações do estado de saúde dos bebês. A técnica de enfermagem informa a uma mãe sobre a quantidade de leite e o ganho de peso gradativo do seu filho.

Diante dos relatos citados e das observações feitas pode ser afirmado que os profissionais buscam acolher os pais que chegam na unidade para visitar seus filhos, passando todo o quadro de saúde dos mesmos, no sentido de tranquilizá-los.

No teste de associação livre de palavras apareceu nos elementos de contraste o termo acolhimento, com frequência de 4 e ordem de importância de 1,750. Pode-se inferir, então, que essa forma de receber os pais na unidade, passando todas as informações necessárias é uma maneira de acolhê-los da melhor forma possível.

\section{Uso de linguagem acessível}

A terceira categoria apreendeu as representações dos profissionais acerca do uso de uma linguagem acessível aos pais dos prematuros, conforme as falas adotadas.

"De acordo o nível de conhecimento a gente vai mudando a linguagem. Se é uma pessoa que requer que a minha linguagem seja a mais simples possível, eu tenho que tentar explicar da forma mais simples pra ela entender, até porque o público que a gente recebe aqui é um público mais simples, a gente precisa até ter um cuidado no falar, às vezes você fala uma coisa que não é nada demais e se torna gritante na cabeça dele porque ele não entende o que é aquilo" (E4).

"Explico pra ela com uma linguagem simples, acessível, que ela compreenda que se o bebê tá com CPAP ele tá precisando de menos ajuda em reação ao que está entubado" (E14).

A observação sistemática, também, constatou essa adequação da linguagem à clientela: A médica passa os boletins com linguagem acessível às mães. A fisioterapeuta explica à mãe o procedimento que estava realizando com o bebê de uma maneira que ela consiga entender.

No teste de associação livre de palavras também apareceu o termo linguagem acessível nos elementos de contraste, com uma frequência de 4 e ordem de importância de 2,750. Assim, pode ser destacada a importância de compreender o nível de conhecimento de cada pessoa e, então, usar palavras adequadas durante as orientações e ensinamentos diários.

\section{Orientações no momento da alta do bebê}

A quarta categoria vem retratar as representações dos profissionais a respeito das orientações no momento da alta hospitalar.

"As orientações no dia da alta, primeiro são todas as solicitações de exames que o médico deixa, a gente vai orientar, como que ele vai marcar, orienta quanto às vacinas, orienta quanto o aleitamento, orienta quanto aos cuidados que tem que ter com o prematuro a respeito das viroses, do contato com outras pessoas, das visitas, do aleitamento, do leite artificial pra ter cuidado até porque tem alguns bebês que saem com prescrição de leite artificial porque a mãe já não tem mais leite devido todas as circunstâncias aqui que acabou secando o leite, a gente faz todas essas orientações" (E4). 
Durante a observação sistemática também foi observado que essas orientações são realizadas no momento da alta hospitalar. A médica chega para dar alta a um bebê e conversa com a mãe sobre o acompanhamento profissional que o bebê vai necessitar após a alta hospitalar; em seguida a enfermeira e a fisioterapeuta, também, dão orientações a essa mãe.

No teste de associação livre de palavras também emergiu a palavra orientações, que aparece nos elementos da $1^{\underline{a}}$ periferia com uma frequência de 22 e ordem de importância de 3,227 . É gratificante ver que a equipe de saúde tem a preocupação de orientar os pais durante o momento da alta hospitalar, uma vez que uma mãe bem orientada proporciona mais tranquilidade e segurança durante o cuidado domiciliar.

\title{
Fornecimento de um folder educativo no momento da alta do bebê
}

A quinta e última categoria mostrou que as representações dos profissionais apreendidas foram acerca do fornecimento de um folder com informações educativas no momento da alta do bebê. Algumas falas retratam essas representações.

\author{
"Já existe um folder pronto que as mães já saem com determinadas \\ orientações” (E2).
}

"Temos um folder, que além do que a gente enfatiza a gente dá pra pessoa ler em casa e se tiver dúvida volta ali naquela leitura” (E4).

"A gente tem umas orientações de alta pro pré-termo e pro pós-termo, são orientações diferentes. Nessas orientações a gente tem tudo que se deve evitar, tudo que se deve fazer em casa em formato de folder. Então a mãe sai daqui a gente lê, o médico lê um por um na alta do paciente, e é reforçado pela equipe de enfermagem" (E5).

Observou-se que, além das orientações verbais, a equipe de saúde entrega um folder com informações importantes sobre o cuidado com o bebê em casa para que as mães consigam ter mais confiança durante ao cuidar dos seus filhos. Com o objetivo de preparar as mães de forma mais eficaz para a alta hospitalar, os profissionais entrevistados fornecem um folder educativo com informações específicas a respeito da amamentação/alimentação, cuidado com as mamas, ordenha de leite, troca de fraldas, assaduras, banho, controle de temperatura, roupas do bebê, posicionamento, prevenção de infecções, sono, banho de sol, cordão umbilical, transporte do bebê e necessidade de vitaminas.

\section{DISCUSSÃO}

A relação entre práticas e representações é recíproca nesse estudo, uma vez que as representações apreendidas condizem com as práticas observadas e relatadas nas entrevistas. Assim, as representações são gerenciadoras de suas práticas, visto que os pensamentos são os guias para as suas ações e assegura que a memória coletiva é necessária para manter ou justificar a identidade, a existência ou a prática dos grupos (ABRIC JC, 2011; WOLTER RP e SÁ CP, 2013).

Foi constatado, diante dos resultados apresentados, que os profissionais buscam sempre passar explicações específicas sobre os procedimentos realizados nos bebês, visto que os pais se encontram fragilizados e esta pode ser uma forma de tranquilizá-los. Um estudo realizado em 2016, sobre a assistência do enfermeiro ao prematuro em UTIN, destacou que os enfermeiros realizam atividades voltadas para orientações aos pais, tanto quanto ao cuidado com o RN sobre o risco de infecção, quanto aos procedimentos que estão sendo realizados nos bebês durante a hospitalização (RIBEIRO JF, et al., 2016)

O impacto dessa hospitalização gera sentimentos ambivalentes, onde normalmente os sentimentos negativos possuem predominância. O papel dos pais fica comprometido devido a hospitalização e eles se sentem impotentes diante das necessidades do bebê, os quais, desencadeiam acontecimentos marcantes para a vida familiar, necessitando de cuidados globais e individualizados (FERNANDES NGV e SILVA EMB, 2015). 
Com isso, existe uma necessidade de acolhimento dos pais pelos profissionais envolvidos, destacando a importância do compartilhamento de informações e saberes profissionais com a família, pois o contato inicial dos pais com a UTIN se encontra rodeado de expectativas e angústias (SOARES LG, et al., 2019).

Os pais, ao observarem as alterações hemodinâmicas, principalmente quando existe agravamento do quadro clínico do bebê, percebem a fragilidade do seu filho, sendo importante que os profissionais estejam preparados para reconhecer estes pais como vulneráveis, proporcionando-lhes segurança, afetividade, atendimento humanizado e informações precisas sobre o estado de saúde do bebê (FERNANDES NGV e SILVA EMB, 2015).

Assim, o compartilhamento das informações e dos saberes profissionais sobre o quadro clínico do bebê, somadas às orientações a respeito do sono/repouso, evolução do padrão respiratório, nutrição, eliminações e tratamento ofertado, são os maiores anseios da família. Ao terem acesso a essas informações, os pais são capazes de lidar melhor com a situação do adoecimento, como também conseguem enfrentar melhor os momentos de fragilidade (SOARES LG, et al., 2019).

É importante salientar que todas as orientações e informações sobre o quadro clínico do bebê devem ser passadas de acordo com o nível de compreensão dos familiares, devendo ter sempre uma linguagem acessível e de forma simples, resultando em uma assistência mais humanizada e segura para todos os envolvidos (SOUSA FCP, et al., 2017).

Durante o momento da alta hospitalar os familiares também devem receber orientações. Em um estudo publicado em 2017 as mães foram submetidas a um processo de preparo para a alta dos seus filhos prematuros. Durante o tempo em que as mães estavam nas unidades neonatais, eram orientadas acerca de diversos temas, elucidando as dúvidas e estimulando-as a participarem dos cuidados junto a seus filhos (VERONEZ M, et al., 2017).

Torna-se evidente a necessidade de envolver a mãe nos cuidados com seu bebê desde a UTIN, com o auxílio de uma equipe qualificada e de acordo com as especificidades de cada caso, pois é permitida uma preparação para a alta hospitalar de modo a promover uma continuidade entre os cuidados no hospital e em casa, proporcionando bons resultados nos cuidados com o bebê no ambiente domiciliar (LEÃO LCS, et al., 2017).

É importante destacar que os recursos de apoio à educação em saúde, a exemplo do folder educativo, oferecem suporte aos profissionais de saúde na condução das atividades educativas cotidianas, além de ser um material que a população pode consultar a qualquer momento que julgar necessário, reforçando a autonomia e empoderamento desta (SILVA IOAM, et al., 2018).

A falta de materiais educativos para orientação dos cuidadores de crianças que nasceram prematuramente mostra a necessidade do fornecimento de materiais com informações confiáveis, baseadas em evidências, por profissionais de saúde (SANCHES MPC, et al., 2017).

Assim, esse estudo pretende contribuir para o fortalecimento das práticas educativas em saúde capazes de oferecer segurança e habilidade aos pais, pois visam prepará-los para o cuidado necessário ao crescimento e ao desenvolvimento saudável de seu filho.

\section{CONCLUSÃO}

O presente artigo trouxe as representações sociais de profissionais sobre suas práticas educativas em saúde realizadas para mães de prematuros, enquanto seus filhos se encontram hospitalizados, visto que uma educação em saúde efetiva na unidade neonatal favorece o desempenho das mães durante o cuidado do prematuro após a alta hospitalar, dando a elas maior segurança e preparo quando as mesmas estão, em casa, com o filho. Assim, esse estudo pretende colaborar com a criação de políticas educacionais dentro das unidades neonatais, direcionadas para a prática dos profissionais envolvidos, uma vez que uma família bem auxiliada é tão importante quanto a qualidade da assistência proporcionada ao recém-nascido hospitalizado. 


\section{REFERÊNCIAS}

1. ABRIC JC. Pratiques sociales et représentations. 1 ed. Paris: PUF, 2011; 303p.

2. AHMAD A, et al. Socioeconomic and Maternal Risk factors for Pre-Term Births in District Swat. Journal of Saidu Medical College, 2018; 8(2): 166-170.

3. BARDIN L. Análise de Conteúdo. 1 ed. São Paulo: Edições 70, 2011; 229p.

4. BUGS BM, et al. Educative activity for preterm infant mothers as a support to care. Revista de Enfermagem do CentroOeste Mineiro, 2018; 8: 2-9.

5. FERNANDES NGV, SILVA EMB. Vivência dos pais durante a hospitalização do recém-nascido prematuro. Revista de Enfermagem Referência, 2015; 4: 107-115.

6. JODELET D. As representações sociais. 1 ed. Rio de Janeiro: UERJ, 2001; 420p.

7. JÚNIOR AFB, et al. Triangulação: uma ferramenta de validade e confiabilidade. Sinergia, 2016; 20(1): 19-28.

8. LEÃO LCS, et al. From NICU to home: maternal experiences in the preterm baby's predischarge. Psicologia em Estudo, 2017; 22(2): 153-164.

9. MAIA JMA, et al. A relação da família com crianças hospitalizadas na unidade de terapia intensiva neonatal com a equipe de enfermagem. Revista Enfermagem Contemporânea, 2014; 3(2): 154-164.

10. MOSCOVICI S. Representações sociais: investigações em psicologia social. 7 ed. Petrópolis: Vozes, 2010; 404p.

11. QUEIROZ MAC, et al. Social representations of sexuality for the elderly. Revista Brasileira de Enfermagem, 2015; 68(4): 662-667.

12. RIBEIRO JF, et al. Fatores favoráveis e desfavoráveis à educação em saúde na alta hospitalar do recém-nascido. Educación Física y Deportes, 2015; 19(202): 1-1.

13. RIBEIRO JF, et al. O prematuro em unidade de terapia intensiva neonatal: a assistência do enfermeiro. Revista de Enfermagem UFPE, 2016; 10(10): 3833-3841.

14. SANCHES SPC, et al. Avaliação de materiais educativos para o cuidado e a promoção do desenvolvimento de crianças nascidas prematuras. Revista da Sociedade Brasileira de Enfermeiros Pediatras, 2017; 17(2): 76-82.

15. SILVA IOAM, et al. Booklet on premature infants as educational technology for the family: quasi-experimental study. Acta Paulista de Enfermagem, 2018; 31(4): 334-341.

16. SOARES LG, et al. Percepção das famílias sobre o acolhimento no contexto neonatal durante um processo de intervenção. Revista Cuidado é Fundamental, 2019; 11(1): 147-153.

17. SOUSA FCP, et al. Family participation in patient safety in neonatal units from the nursing perspective. Texto \& Contexto Enfermagem, 2017; 26(3): 1-8.

18. VERONEZ M, et al. Experience of mothers of premature babies from birth to discharge: notes of field journals. Revista Gaúcha de Enfermagem, 2017; 38(2): 1-8.

19. WHO. WORLD HEALTH ORGANIZATION. Preterm birth Fact. 2018. Disponível em: https://www.who.int/newsroom/fact-sheets/detail/preterm-birth: Acessado em: 20 mar. 2019.

20. WOLTER RP, SÁ CP. As relações entre representações e práticas: o caminho esquecido. Revista Internacional de Ciencias Sociales y Humanidades, 2013; 23(1): 87-105. 